\title{
Identification of Mycobacterium smegmatis in Bovine Mastitis
}

\author{
Franciele Maboni Siqueira, Cassiane Elisabete Lopes, Gustavo Geraldo Snell \& Marcos José Pereira Gomes
}

\begin{abstract}
Background: Rapidly growing mycobacteria (RGM) are ubiquitous in the environment, can be isolated from soil and water, and demonstrate visible growth on culture media within seven days. Mycobacterium smegmatis is an acid-alcohol fast bacterium, which belong to RGM group. The diagnosis of $M$. smegmatis infections may be quite difficult by conventional methods; therefore, biochemistry associated to nucleic acid-based approaches provided fast and accurate identification. Although this specie may be associated to animals and humans infections, there is few cases description. Nontuberculous mycobacterial bovine mastitis is uncommon, and bovine mastitis by M. smegmatis has been reported but non-confirmed case once in the past. This paper reports $M$. smegmatis recovered from a cattle with relapsing pyogranulomatous mastitis. Case: Milk samples from an adult Holstein cow showing relapsing pyogranulomatous mastitis history and by pronounced glandular hardening were cultivated and analyzed accordingly to standard milk cultivation protocols. The animal had been subject to several intramammary and parenteral antibiotic therapies protocols without adequate response. After 48 $\mathrm{h}$ incubation, a slow and sparse growth of slightly pigmented, shiny and smooth colonies was observed on the blood agar plate. The bacterium isolated was named as strain 55/08. The morphological and biochemical profile were tested, and the ability of the isolate to grow at Lowenstein-Jensen slants was confirmed. The isolated have showed positive reaction to catalase, glucose, sucrose, mannitol and nitrate. The pigment formation was observed for 14 days incubation, and the colonies produce pigment after prolonged time. Gram and Ziehl-Neelsen staining revealed poorly pigmented, irregular, slender Gram-positive and acid fast rods. The staining and biochemical profile showed closed isolated relationship to $M$. smegmatis. A discriminatory identification based in the 16S rRNA gene sequence analysis was performed. The total DNA from the strain 55/08 was extracted and the partial 16S rRNA sequence was amplified, using prokaryotic universal primer pairs and the extract DNA as template, by PCR assay following the purification and sequencing of the amplicons. A total of 1,443 nucleotides form consensus sequence were alignment to $M$. smegmatis and other mycobacteria 16S rRNA available sequences. The sequence analysis confirmed the M. smegmatis identification as etiological agent of bovine relapsing pyogranulomatous mastitis. M. smegmatis strain 55/08 partial 16S rRNA gene sequence was submitted to GenBank. The phylogenetic relationship of the strain 55/08 with other mycobacteria was performed in order to confirm the identification of the isolate as M. smegmatis.

Discussion: Nontuberculous mycobacteria are uncommon causes of bovine mastitis. Some old reports have described $M$. smegmatis as etiological agent of mastitis, but without definitive diagnostic. M. smegmatis mammary quarter introduction may be related to the repeated intramammary treatment protocols, because this mycobacteria is related to environmental infections. The relapsing pyogranulomatous mastitis infection could be associated to other bacteria species. However, the phenotypic and molecular characterization which was performed demonstrated the accurate identification of the isolated as M. smegmatis. Milk contaminated by M. smegmatis may be a potential infection source for human and other animal species. This report reinforces the need to optimize quality programs and laboratorial diagnosis to further the accurate microorganism identification in milk samples.
\end{abstract}

Keywords: RGM mycobacteria, relapsing mastitis, M. smegmatis, molecular identification. 


\section{INTRODUCTION}

Mycobacteria are aerobic, non-motile, Grampositive rods with a cell wall rich in mycolic acids and mycosides [8]. Rapidly growing mycobacteria (RGM) are ubiquitous in the environment, can be isolated from soil and water, and demonstrate visible growth on culture media within seven days. RGM comprise the following groups: Mycobacterium chelonae-abscessus, Mycobacterium fortuitum and Mycobacterium smegmatis [8].

Mycobacteria from $M$. smegmatis group are occasionally associated to animals and humans infections $[2,8]$. M. smegmatis was previously associated to canine dermatitis and panniculitis [2], bovine mastitis [10], human wound infections, especially post traumatic soft-tissue infections $[1,3,9]$. Therefore, $M$. smegmatis has a zoonotic potential.

The diagnosis of $M$. smegmatis infections may be quite difficult by conventional methods [6]; therefore, biochemistry associated to nucleic acid-based approaches provided fast and accurate identification. Additionally, partial 16S rRNA gene sequence analysis has been used to describe the phylogenetic relationships among mycobacterial species [11].

Nontuberculous mycobacterial bovine mastitis is uncommon, but it has been report that the $M$. smegmatis group species may cause clinical mastitis in sheep and dairy cows $[4,7,14]$. The aim of this study was to describe an unusual case of bovine relapsing pyogranulomatous mastitis caused by $M$. smegmatis specie.

\section{CASE}

An adult Holstein cow belonging to a herd originally located in the south of Brazil, showed mastitis signs of pronounced glandular hardening. The animal was subject to several intramammary (aminoglycoside and b-lactam) and parenteral (tetracyclines) antibiotic therapies protocols. However, no adequate response was observed, and milk bacteriological test was requested.

Milk samples from the four quarters were submitted to a standard milk culture (blood agar and MacConkey medium at aerobic incubation at $37^{\circ} \mathrm{C}$ ), and routine biochemical tests were performed. After $48 \mathrm{~h}$ incubation, a slow and sparse growth of lightly pigmented, shiny and smooth colonies ranging from 1 to $2 \mathrm{~mm}$ was observed on the blood agar plate (Figure 1A), and no growth was observed on the MacConkey medium. The mycobacterium isolated was named as strain 55/08.

The morphological and biochemical profile were tested, and the ability of the isolate to grow at Lowenstein-Jensen slants was confirmed. The pigment formation was observed for 14 days incubation, and the colonies produce pigment after prolonged time.

Gram and Ziehl-Neelsen staining revealed poorly pigmented, irregular, slender Gram-positive and acid-fast rods (Figure 1B). Biochemical characterization of the isolate showed that it was catalase, glucose, sucrose, mannitol and nitrate positive reduction and was negative for maltose, CAMP test, oxidase and aesculin. The isolate has in vitro similar pattern to M. smegmatis as similar previously reported [5,8].

Although phenotypic characteristics led to $M$. smegmatis, we performed a discriminatory identification based in the 16S rRNA gene sequence analysis. The total DNA from the strain 55/08 was extracted by lysis with cetyltrimethylammonium bromide (CTAB) and isolation by the addition of phenol: chloroform according to Sambrook \& Russell [12]. PCR assay was performed to amplify a partial $16 \mathrm{~S}$ rRNA sequence using prokaryotic universal oligonucleotides 27F (5'-AGAGTTTGATCMTGGCTCAG-3') and 1492R (5'-GGTTACCTTGTTACGACTT-3') positions.

PCRs assay included 1 U GoTaq DNA polymerase $^{1}, 5 \mathrm{X}$ of GoTaq buffer, $1 \mathrm{mM}$ of each deoxynucleotide triphosphate, $10 \mathrm{pmol}$ of each primer and $30 \mathrm{ng}$ of the total DNA previously extracted in a final volume of $25 \mu \mathrm{L}$. The PCR conditions were: 1 cycle at $94^{\circ} \mathrm{C}$ for $5 \mathrm{~min}$ followed by 35 cycles of $94^{\circ} \mathrm{C}$ for $30 \mathrm{~s} ; 55^{\circ} \mathrm{C}$ for $30 \mathrm{~s}$ and $72^{\circ} \mathrm{C}$ for $1.2 \mathrm{~min}$. The negative control was prepared in parallel, differing from the other reactions by the absence of genomic DNA. The final extension step was at $72^{\circ} \mathrm{C}$ for $10 \mathrm{~min}$. Reaction products were analyzed in $1 \%$ agarose gels.

The amplicons were precipitated by transfer RNA (tRNA) ${ }^{2}$ and sequenced in an automatic sequencer ABI-PRISM 3500 Genetic Analyzer ${ }^{3}$. DNA fragments from overlapping strands generated a consensus sequence. A total of 1,443 nucleotides in the consensus sequence were alignment to $M$. smegmatis and other mycobacteria 16S rRNA sequence from GenBank (NCBI) database to determine similarity. M. smegmatis strain 55/08 partial 16S rRNA gene sequence was submitted to GenBank and it is available by accession number: KX298207.1. 


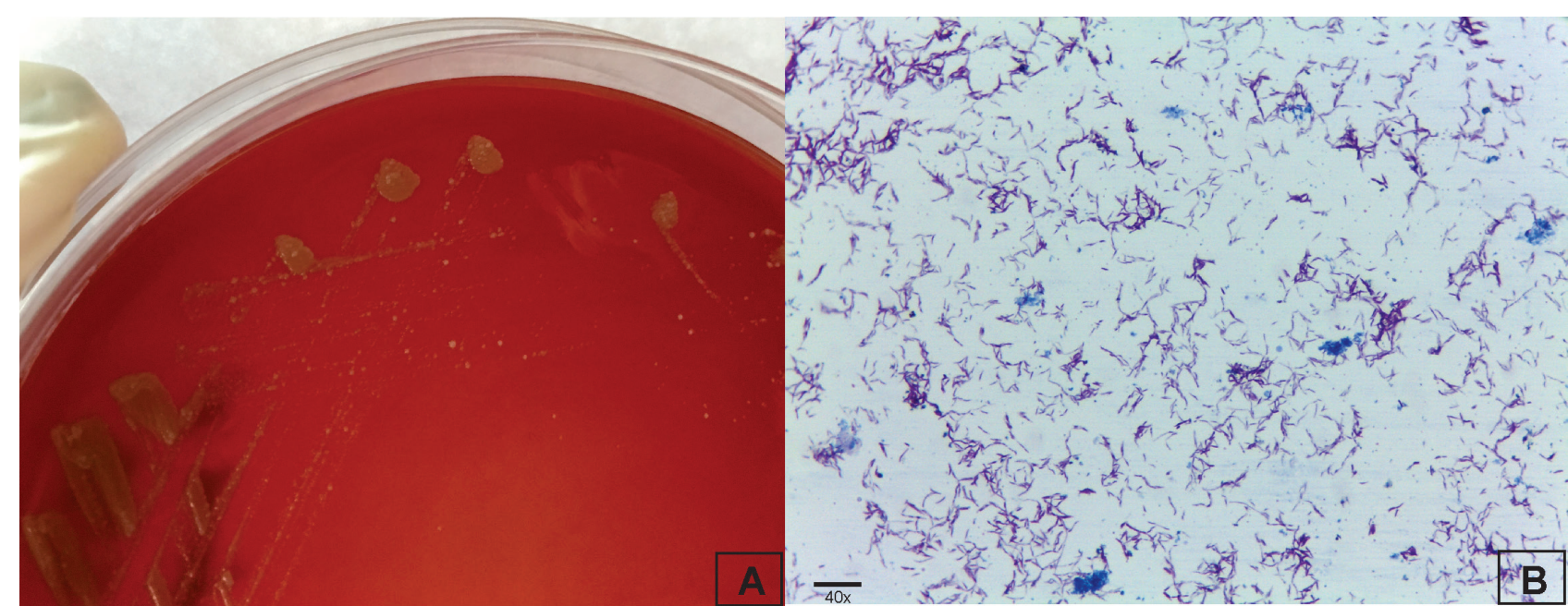

Figure 1. Mycobacterium smegmatis phenotypic characterization. A- Blood agar cultivation showing sparse growth of lightly pigmented, shiny and smooth colonies. B- Ziehl-Neelsen staining with poorly pigmented, irregular, slender acid-fast rods [magnification: 40x].

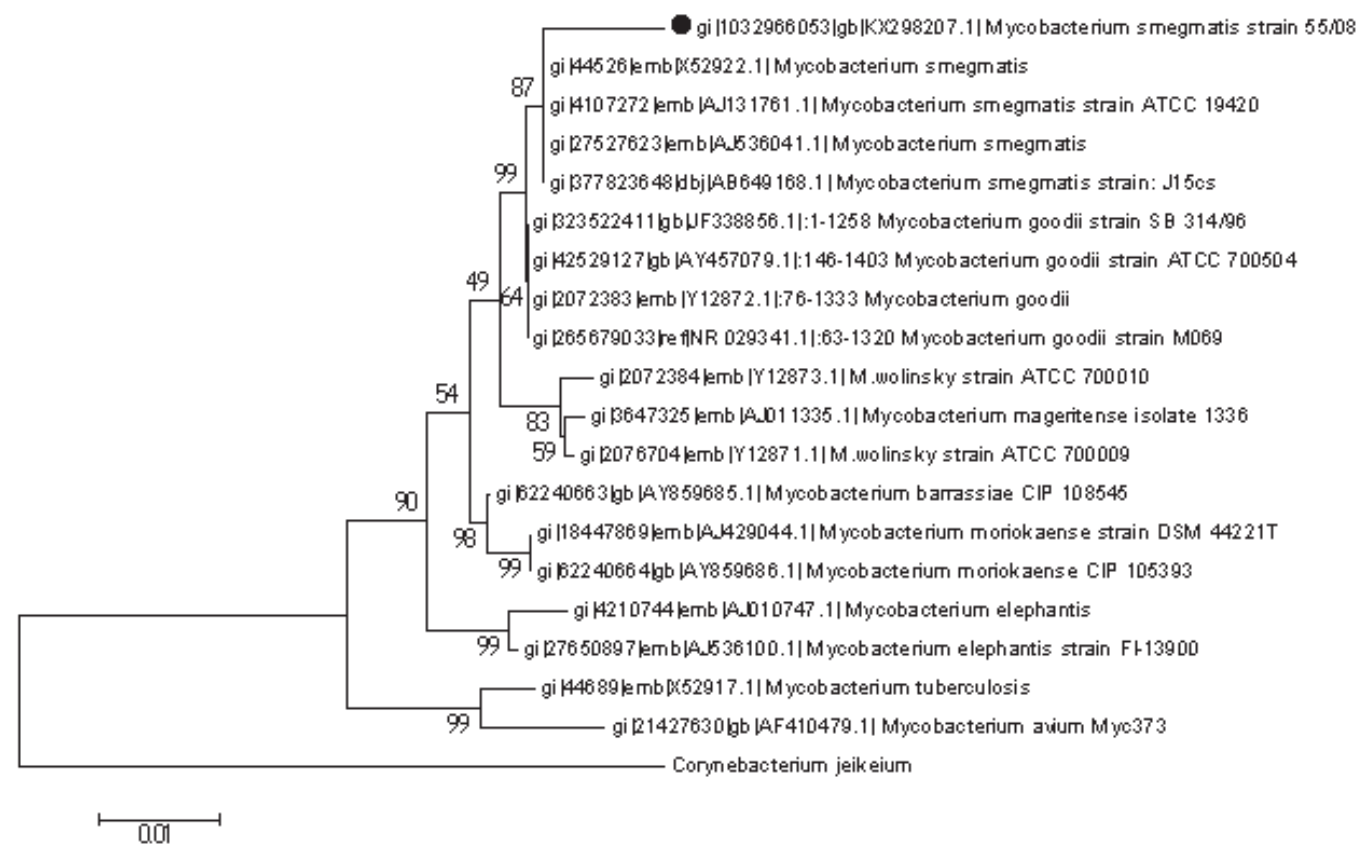

Figure 2. Evolutionary relationships of Mycobacterium smegmatis 55/08 strain. Phylogenetic analysis based on 16S rRNA partial sequences showing the homology between the 16S rRNA gene sequence of $M$. smegmatis 55/08 (circle detached) and other 16S rRNA gene sequences of M. smegmatis and Mycobacterium sp. Evolutionary analyses were inferred using the Neighbor-Joining method and conducted in MEGA5 software. The percentage of replicate trees in which the associated taxa clustered together in the bootstrap test (2,000 replicates) is shown next to each branch. Corynebacterium jeikeium was used as outgroup.

The phylogenetic relationship of the strain $55 / 08$ with other mycobacteria was performed in order to confirm the identification of the isolate as $M$. smegmatis (Figure 2). Neighbour-joining method was used, and the evolutionary distances and the pairwise deletion of gaps were calculated by the p-distance implementation in the MEGA v.5.2.2 software [13].

\section{DISCUSSION}

Nontuberculous mycobacteria are uncommon causes of bovine mastitis. Some old reports have described $M$. smegmatis as etiological agent of mastitis, but without definitive diagnostic. M. smegmatis mammary quarter introduction may be related to the repeated intramammary treatment protocols, 
because this mycobacteria is related to environmental infections. The relapsing pyogranulomatous mastitis infection could be associated to other bacteria species [7,14]. However, the phenotypic and molecular characterization which was performed demonstrated the accurate identification of the isolated as M. smegmatis.

Here we described a rare bovine mastitis caused by $M$. smegmatis. Our report reinforces the need to optimize quality programs and laboratorial diagnosis to further the accurate microorganism identification in milk samples. Moreover, milk M. smegmatis contamination may be a potential infection source for human and other animal species.

\section{MANUFACTURERS \\ ${ }^{1}$ Promega. Madison, WI, USA \\ ${ }^{2}$ Invitrogen ${ }^{\mathrm{TM}}$. São Paulo, SP, Brazil. \\ ${ }^{3}$ Applied Biosystems Inc. Foster City, CA, USA.}

Declaration of interest. The authors report no conflicts of interest. The authors alone are responsible for the content and writing of the paper.

\section{REFERENCES}

1 Best C.A. \& Best T. 2009. Mycobacterium Smegmatis Infection of the Hand. Hand. 4(2): 165-166.

2 Barletta R.G. \& Steffen D.J. 2013. Mycobacterium. In: McVey D.S., Kennedy M. \& Chengappa M.M. (Eds). Veterinary Microbiology. 3rd edn. Hoboken: Wiley-Blackwell, pp.277-286.

3 Bohsali A., Abdalla H., Velmurugan K. \& Briken V. 2010. The non-pathogenic mycobacteria M. smegmatis and $M$. fortuitum induce rapid host cell apoptosis via a caspase-3 and TNF dependent pathway. BMC Microbiology. 10: 237.

4 Franco M.M.J., Paes A.C., Ribeiro M.G., Pantoja J.C.F., Santos A.C.B., Miyata M., Leite C.Q.F., Motta R.G. \& Listoni F.J.P. 2013. Occurrence of mycobacteria in bovine milk samples from both individual and collective bulk tanks at farms and informal markets in the southeast region of São Paulo, Brazil. BMC Veterinary Research. 9: 85.

5 Hartmans S., De Bont J.A.M. \& Stackebrandt E. 2006. The genus Mycobacterium - nonmedical. Prokaryotes. 3: 889-918.

6 Lemarie S.L. 1999. Mycobacterial dermatitis. Veterinary Clinics of North America: Small Animal Practice. 29: 12911301.

7 Machado G., Gressler L.T., Siqueira F.M., Balzan C., Brum J.S. \& Vargas A.P.C. 2015. Bovine pyogranulomatous mastitis caused by Mycobacterium goodii. JMM Case Reports. 2: e004150.

8 Quinn P.J., Carter M.E., Markey B. \& Carter G.R. 1994. Mycobacterium species. In: Quinn P.J., Carter M.E., Markey B. \& Carter G.R. (Eds). Clinical Veterinary Microbiology. St. Louis: Mosby- Elsevier Limited, pp.156-169.

9 Pennekamp A., Pfyffer G.E., Wüest J., George C.A. \& Ruef C. 1997. Mycobacterium smegmatis infection in a healthy woman following a facelift: case report and review of the literature. Annuals Plastic Surgery. 39(1): 80-83.

10 Richardson A. 1970. Bovine mastitis associated with Mycobacterium smegmatis and an untypable Mycobacterium. Veterinary Record. 86(17): 497-498.

11 Rogall T., Wolters T., Flohr T. \& Böttger E.C. 1990. Towards a phylogeny and definition of species at the molecular level within the genus Mycobacterium. International Journal System Bacteriology. 40: 323-330.

12 Sambrook J. \& Russell D.W. 2001. Preparation and analysis of Prokaryotic genomic DNA. In: Molecular Cloning. 3rd edn. v.1. New York: Cold Spring Habor Laboratory Press, p.6.62.

13 Tamura K., Peterson D., Peterson N., Stecher G., Nei M. \& Kumar S. 2011. MEGA5: Molecular Evolutionary Genetics Analysis using Maximum Likelihood, Evolutionary Distance, and Maximum Parsimony Methods. Molecular Biological and Evolution. 28: 2731-2739.

14 Thomson J.R., Mollison N. \& Matthews K.P. 1988. An investigation of mastitis due to S. agalactiae, S. uberis and M. smegmatis in a dairy herd. Veterinary Records. 122: 271-274. 